\title{
Spectral linewidth of autonomous and injection-locked flux-flow oscillators
}

\author{
Koshelets, V. P.; Shchukin, A.; Lapytskaya, I. L.; Mygind, Jesper
}

Published in:

Physical Review B

Link to article, DOI:

10.1103/PhysRevB.51.6536

Publication date:

1995

Document Version

Publisher's PDF, also known as Version of record

Link back to DTU Orbit

Citation (APA):

Koshelets, V.P. ., Shchukin, A., Lapytskaya, I. L., \& Mygind, J. (1995). Spectral linewidth of autonomous and injection-locked flux-flow oscillators. Physical Review B, 51(10), 6536-6541.

https://doi.org/10.1103/PhysRevB.51.6536

\section{General rights}

Copyright and moral rights for the publications made accessible in the public portal are retained by the authors and/or other copyright owners and it is a condition of accessing publications that users recognise and abide by the legal requirements associated with these rights.

- Users may download and print one copy of any publication from the public portal for the purpose of private study or research.

- You may not further distribute the material or use it for any profit-making activity or commercial gain

- You may freely distribute the URL identifying the publication in the public portal

If you believe that this document breaches copyright please contact us providing details, and we will remove access to the work immediately and investigate your claim. 


\title{
Spectral linewidth of autonomous and injection-locked flux-flow oscillators
}

\author{
V. P. Koshelets, A. Shchukin, and I. L. Lapytskaya \\ Institute of Radio Engineering and Electronics, Russian Academy of Sciences, Moscow, Russia
}

\section{J. Mygind}

Physics Department, Technical University of Denmark, DK-2800 Lyngby, Denmark

(Received 22 February 1994; revised manuscript received 15 November 1994)

\begin{abstract}
Oscillators based on unidirectional viscous flow of magnetic flux quanta in long Josephson tunnel junctions with high damping have been experimentally investigated by harmonic mixing and frequency multiplication at frequencies up to $450 \mathrm{GHz}$. An integral spectral linewidth of two fluxflow oscillators (FFO) as low as $750 \mathrm{kHz}$ was found at $280 \mathrm{GHz}$. Presently no theory exists for the linewidth of the FFO. Above a few $\mathrm{MHz}$ the experimental linewidth scales with the square of the dynamic resistance of the dc $I-V$ curve as found for short Josephson junctions and oscillators based on resonant motion of fluxons in under-damped long Josephson tunnel junctions. Based on calculations assuming wideband thermal noise, we find that the effective temperature of the FFO appears to be much higher than its physical temperature. Different models are discussed in order to explain this discrepancy. Finally harmonic phase locking of a FFO at $355 \mathrm{GHz}$ is demonstrated.
\end{abstract}

\section{INTRODUCTION}

The unidirectional and viscous flows of magnetic flux quanta in a long (length $L \gg \lambda_{J}$, the Josephson penetration depth) Josephson tunnel junction with high damping ${ }^{1}$ have recently been successfully used in the development of local oscillators (LO's) for fully superconducting integrated submillimeter wave superconductorinsulator-superconductor (SIS) receivers. ${ }^{2-6}$ The frequency of this so-called flux-flow oscillator (FFO) can be tuned over a wide frequency range, limited only by the superconductor gap frequency. Moreover, the FFO provides sufficient output power to pump a SIS array mixer detector. Preliminary FFO spectral linewidth measurements ${ }^{2,5,6}$ have demonstrated encouraging values $\left[130 \mathrm{kHz}\right.$ at $70 \mathrm{GHz},{ }^{2}$ about $1 \mathrm{MHz}$ at $140 \mathrm{GHz},{ }^{5}$ and $2.1 \mathrm{MHz}$ at $320 \mathrm{GHz}$ (Ref. 6)]. One-dimensional arrays of phase-locked small Josephson junctions already have been shown to deliver ample power for pumping a SIS detector, ${ }^{7-9}$ and recently the two-dimensional array has attracted much interest. ${ }^{10,11}$ In this paper we report on experimental measurements of the FFO's linewidth as function of applied dc bias current and magnetic field, both with two autonomous FFO's as well as with one of the FFO's phase locked by injection to harmonics of a narrow-band external microwave source.

\section{EXPERIMENTAL SYSTEM}

A sketch and a simplified equivalent diagram of the on-chip integrated circuit is shown in Fig. 1. The circuit comprises two identical FFO's [FFO1 (10) and FFO2 (11), length $L=200 \mu \mathrm{m}$, and width $W=1.5 \mu \mathrm{m}]$, a SIS mixer array detector with a capacitance-tune-out circuit
(14, area $\left.S=1.3 \times 1.3 \mu \mathrm{m}^{2}\right)$, three impedance matching transformers $(9,12$, and 13$)$, and a fin-line antenna (8). The tuning-out inductances for the two SIS junction (parallel dc biased ${ }^{12}$ ) array (14) and the transformers (12 and 13) were designed for optimum performance at 450

(a)

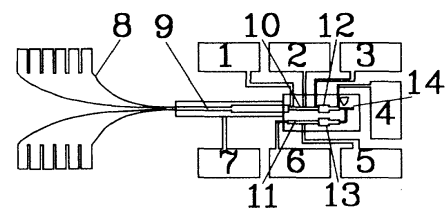

(b)

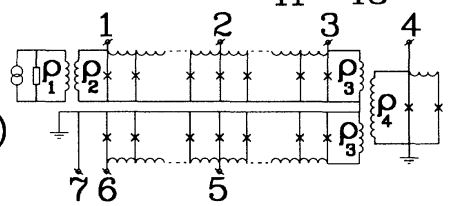

(c)

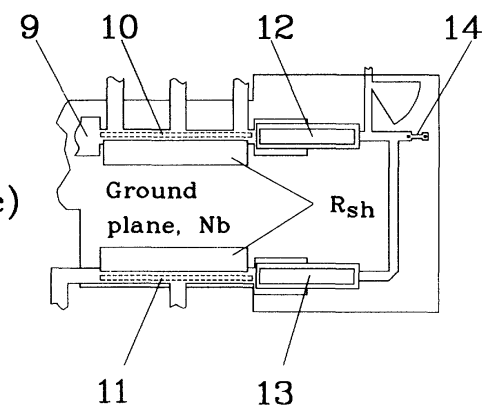

FIG. 1. Schematic drawing (a), simplified equivalent diagram (b), and (c) blowup of the central part of the experimental on-chip integrated circuit. 1-7, contact pads; 8 , fin-line antenna; 9, 12, 13, Chebyshev impedance matching transformers; 10, FFO1; 11, FFO2; 14, two-junction SIS array with tuning-out circuit. 
$\mathrm{GHz}$. The integrated circuits have been fabricated with a technique developed for producing SIS mixer elements and rapid single-flux quantum (RSFQ) digital devices. ${ }^{13}$ Details of the circuit design and experimental setup will be described elsewhere. ${ }^{14}$ The high-quality $\mathrm{Nb}-\mathrm{AlO}_{x}-\mathrm{Nb}$ tunnel junctions have a critical current density $j_{c}=8$ $\mathrm{kA} / \mathrm{cm}^{2}$ and a Josephson penetration depth $\lambda_{j}=4 \mu \mathrm{m}$. The product of the normal state resistance and the junction area, $R_{n} \times S$, is $25 \Omega \mu \mathrm{m}^{2}$.

\section{INTEGRAL LINEWIDTH OF TWO AUTONOMOUS FFO's}

In the first experiments the signals from two freerunning autonomous FFO's were mixed in the small SIS array detector. The frequencies of the FFO's could be individually tuned by adjusting the dc bias current and/or the applied dc magnetic field. Bias currents could be injected either to the ends of the long junctions or evenly distributed along the sides as shown in Fig. 1(c). Typically a homogeneous magnetic field from an external coil was first used to optimize the operational conditions for FFO2 and subsequently an additional field from a control line positioned near FFO1 was used to fine tune the frequency of FFO1 so that their difference frequency was within the band of the intermediate frequency (IF) amplifier connected to the SIS mixer array. The room temperature IF amplifier (center frequency $f_{\mathrm{IF}}=1.5 \mathrm{GHz}$, bandwidth $\Delta B_{\mathrm{IF}}=600 \mathrm{MHz}$ ) had an effective noise temperature of $100 \mathrm{~K}$ referred to the SIS junction array.

With this setup we have detected the mixed signal up to a FFO frequency of $450 \mathrm{GHz}$. As shown in Fig. 2 the total measured (integral $3 \mathrm{~dB}$, half power, full width) linewidth of the two FFO's was as low as $750 \mathrm{kHz}$ at 280 $\mathrm{GHz}$. In order to minimize the influence from external disturbances (fluctuations in temperature and dc current bias conditions) the measurements were made at $T=2.0$ $\mathrm{K}$ (below the $\lambda$ point of liquid helium) on one of the sharp (low dynamic resistance) steps present in the $I-V$ curves of the FFO's. In the spectrum analyzer following the IF amplifier the final linewidth result was found as an average of 100 spectrograms (total sweep time $50 \mathrm{~ms}$ $\times 100)$.

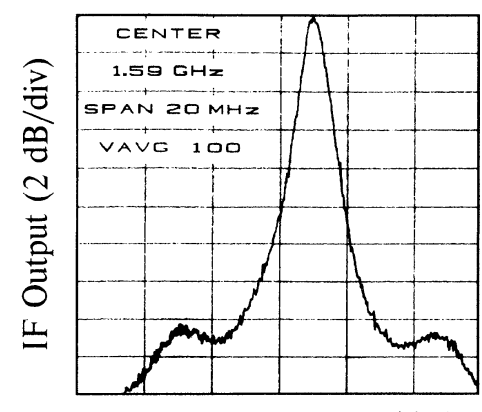

Frequency $(2 \mathrm{MHz} / \mathrm{div})$

FIG. 2. Integral linewidth measured by beating two autonomous FFO's at $280 \mathrm{GHz}$ (see text). $T=2 \mathrm{~K}$.
The results confirm the rather similar experiments done by Zhang et al. ${ }^{6}$ The inherent problem with this kind of measurement is that one may find an unrealistic small integral linewidth because most external disturbances are common to the two FFO's placed on the same chip. In fact, if the FFO's were identical only signals (e.g., from uncorrelated internal noise sources) that are noncommon mode to the FFO's contribute to the integral linewidth observed as the average of the fluctuations of the difference frequency. As an example the integral method may lead to an erroneous interpretation of the linewidth measurements if the two FFO's due to mutual nonlinear interactions perturb each other over a frequency range larger than the IF frequency.

With the given voltage resolution the steps in the dc $I-V$ curves at which the narrow linewidth shown in Fig. 2 was obtained were too small to allow for a well-defined measurement of the dynamic resistance. Also it was not possible to see whether the steps originate from a locking of the two FFO's to internal resonant modes or to an external resonator. From varying the dc current and/or the magnetic field around the bias points it was clear (no mutually induced Shapiro steps) that they did not strongly influence each other in the vicinity of $280 \mathrm{GHz}$.

\section{FFO AS HARMONIC MULTIPLIER}

In order to overcome the above-mentioned problems and to measure the correct linewidth of a single FFO at different frequencies, e.g., by varying the FFO frequency when biasing along the flux-flow step (FFS or velocity-matched step $^{1}$ ) in the $I-V$ curve we used another high-frequency scheme. A signal from an external microwave source (a frequency locked Gunn oscillator with frequency $f_{G}=56-74 \mathrm{GHz}$ and linewidth $\Delta f_{G} \leq 80 \mathrm{kHz}$ ) was introduced in the integrated circuit through the fin-line antenna ( 8 in Fig. 1) and the three-step Chebyshev stepped stripline transformer (9 in Fig. 1) both designed for $70 \mathrm{GHz}$ center frequency. As a result rf-induced Shapiro steps with "usual" voltage spacing $\Delta V=h f /(2 e)$ could be generated in the $I-V$ curve of FFO1 connected to the antenna.

The applied frequency, however, is much lower than the maximum plasma frequency of FFO1, $f_{p 0}>200 \mathrm{GHz}$, and the fundamental microwave signal cannot propagate inside the FFO1 tunnel junction. Nevertheless, from the rf-induced steps in the $I-V$ curve of the SIS array at voltages corresponding to higher harmonics of the applied signal it was inferred that higher harmonic signals (generated by the nonlinearity of the FFO1) reached the SIS detector. Curve $a$ in Fig. 3 shows the unperturbed dc $I-V$ curve of the SIS mixer array. For small levels of applied power the third harmonic signal [frequency $3 f_{G} \approx 220 \mathrm{GHz}$, which roughly coincides with half the design frequency for the transformer (12) and the SIS mixer tuning-out inductance] dominantly affects the detector (see curve $b$ in Fig. 3). At higher power levels (where the critical current of the FFO1 and thus the plasma frequency is suppressed) also a signal at the fundamental frequency of the Gunn oscillator reaches the 


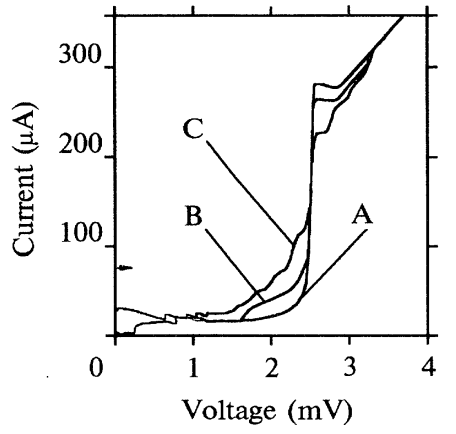

FIG. 3. $I-V$ characteristics of the SIS detector for different levels of the external microwave signal $f_{G}=65.4 \mathrm{GHz}$ applied to the circuit through the fin-line antenna and FFO1 (see text); attenuation (a) $>70 \mathrm{~dB}$ (autonomous $I-V$ curve), (b) $28 \mathrm{~dB}$, and (c) $14 \mathrm{~dB}$.

detector (curve $c$ in Fig. 3). In none of the cases discussed above was dc current applied to the FFO1 and it remained in the zero voltage state.

The observed IF frequency $\left[f_{\mathrm{IF}}\right.$ resulting from the mixing of the $n$th harmonic of the Gunn oscillator with the FFO2 signal (frequency $f_{\mathrm{FFO} 2}$ )] obeys

$$
f_{\mathrm{FFO} 2}=n f_{G} \pm f_{\mathrm{IF}}
$$

and so it is concluded that the FFO1 has successfully been used as a harmonic multiplier. ${ }^{2}$ It was possible to realize harmonic mixing at $n=4,5,6$, and 7 with appropriate Gunn oscillator and FFO2 frequencies. By changing the FFO2 voltage and thereby tuning the FFO2 frequency one can measure both lower and upper sidebands $\left[ \pm f_{\mathrm{IF}}\right.$ in Eq. (1)]. Due to the inserted series of filters and isolators in the waveguide leading to the cryostat we are confident that no higher harmonic signals from the Gunn oscillator can circumvent FFO1 and reach the SIS array directly. Also no detectable influence of the Gunn signal or its harmonics on FFO2 was observed and the FFO2 frequency could be independently and permanently tuned.

\section{LINEWIDTH OF A SINGLE FFO}

The observed dependence of the $3 \mathrm{~dB}$ linewidth of the mixed signal $\Delta f$ on the differential resistance $R_{d}$ of the FFO2 is shown in Fig. 4. The corresponding $I-V$ curve of the FFO2 is depicted in the inset to Fig. 4. The frequency of the FFO2 was changed by more than $15 \mathrm{GHz}$ around $350 \mathrm{GHz}$; the sixth harmonic of the Gunn was used in this case. The contribution from the Gunn oscillator $\left(\Delta f_{G}<\right.$ $80 \mathrm{kHz})$ to the measured linewidth was negligible $(80 \mathrm{kHz}$ $\times 6 \approx 0.5 \mathrm{MHz})$. Actually the linewidth of the Gunn oscillator was rechecked in the frequency-locked mode for the points with smallest FFO linewidths. Accordingly, the measured linewidth of the mixed signal can be attributed to the FFO2 alone. The $R_{d}$ values used in Fig. 4 were derived from the slope of the dc $I-V$ curve in the bias point but when needed a very accurate deter-

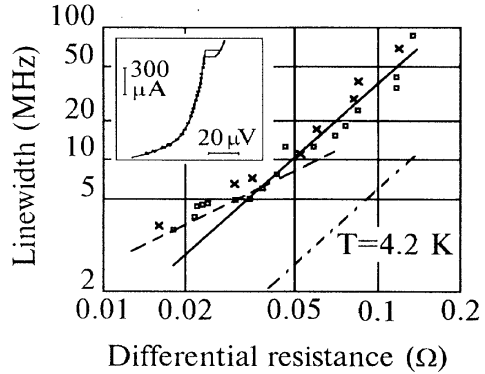

FIG. 4. Dependence of the linewidth $\Delta f$ of FFO2 on the dynamic resistance $R_{d}$ for two runs (indicated as $\times$ and $\square$ ) at $4.2 \mathrm{~K}$. Solid line and dot-dashed line, Eq. (2) with effective temperature $T_{\text {eff }}=32 \mathrm{~K}$ and $4.2 \mathrm{~K}$, respectively; dashed line, Eq. (3) with the amplitude of the low-frequency noise current $I_{f}=0.17 \mu \mathrm{A}$.

mination of the dynamic resistance of the FFS could be made from simultaneous readings of the values of the bias current and the voltage calculated from the observed IF frequency.

One can see from Fig. 4 that the FFO2 linewidth changes proportional to $R_{d}^{2}$ above $5 \mathrm{MHz}$; below this value $\Delta f$ approaches a linear dependence (slope $=1$ ).

Presently, no theory exists for the linewidth of the radiation emitted from a FFO. Both theoretical models for the single Josephson junction ${ }^{15}$ and the lowdamping soliton oscillator ${ }^{16}$ give the same dependence of the linewidth on the differential resistance: $\Delta f \propto R_{d}^{2}$ assuming a wideband $\left(0<f_{\text {noise }} \leq \Delta f\right)$ Nyquist noise spectrum. The linewidth of the high-frequency oscillations in a short Josephson tunnel junction in the limit of small junction current fluctuations is given by ${ }^{15}$

$$
\Delta f_{\mathrm{WB}}=2 \pi \frac{R_{d}^{2}}{\Phi_{0}^{2}} e I_{\mathrm{dc}} \operatorname{coth}\left(\frac{e V_{\mathrm{dc}}}{2 k_{B} T_{\mathrm{eff}}}\right),
$$

where the subindex on $\Delta f_{\mathrm{WB}}$ refers to the wideband noise spectrum with effective temperature $T_{\text {eff }} . V_{\mathrm{dc}}$ and $I_{\mathrm{dc}}$ are the dc voltage and current in the bias point. $\Phi_{0}$ is the flux quantum, $e$ is the electron charge, and $k_{B}$ is Boltzmann's constant.

The best fit (solid line in Fig. 4) to Eq. (2) using the experimental values for $R_{d}, V_{\mathrm{dc}}$, and $I_{\mathrm{dc}}$ is obtained for $T_{\text {eff }}=32 \mathrm{~K}$, which is approximately 8 times the physical temperature $T=4.2 \mathrm{~K}$ (dot-dashed line in Fig. 4). It should be noted that in some experiments with the same sample (for apparently the same experimental conditions) the $\Delta f \propto R_{d}{ }^{2}$ dependence is still observed but a $T_{\text {eff }}$ as large as $90 \mathrm{~K}$ was needed to obtain the best fit. In the original experiments with the linewidth of the lowdamped soliton oscillator ${ }^{16} T_{\text {eff }} \approx 3 T$, this excess noise discrepancy has never been resolved.

The unexpected and aberrant excess noise temperature observed for the FFO may have several origins. The wideband noise (with roll-off frequency higher than 1$5 \mathrm{MHz}$, corresponding to the width of the unperturbed spectral line) will act as thermal noise for the Josephson junction increasing the effective noise temperature. In principle, also any high-frequency signal (e.g., interference from TV transmitters, portable phones, comput- 
ers, etc.) will contribute additional noise. The measurements were performed in a shielded room and only highfrequency signals generated inside the room should influence the measurements. As mentioned all wires connecting to the sample holder and to the chip were carefully hf shielded and filtered.

A likely reason for the large FFO linewidth could be fluctuations in the fluxon velocity when moving along Josephson junction caused by (i) inhomogeneities in the barrier and/or (ii) perturbations imposed by trapped flux in the superconducting films near or inside the junction region. The configuration and dynamics of the trapped flux and thus the noise level might be different from one experiment to another, resulting in the observed changes in the effective noise temperature.

Low-frequency noise with a cutoff frequency much smaller than the width of the spectral line $\left(0 \approx f_{\text {noise }} \ll\right.$ $\Delta f$ ), e.g., from external disturbances (bias supplies, temperature fluctuations, hum, $1 / f$ noise, etc.) may be directly converted and give rise to excess noise. In the experiments a large effort was done in order to minimize extraneous bias noise by filtering of all leads, twisting pairs, use of individual battery supplies, etc. The problem here is merely to find the spectral density of the noise. A low-frequency current noise with amplitude $I_{f}$ will increase the linewidth proportional to $R_{d}$ :

$$
\Delta f_{\mathrm{LF}}=\frac{2}{\Phi_{0}} I_{f} R_{d}
$$

The corresponding dependence for $I_{f}=0.17 \mu \mathrm{A}$ is shown in Fig. 4 by the dashed line fitting the experimental results well for $\Delta f$ below $5 \mathrm{MHz}$. For the data mentioned above with $T_{\text {eff }}=90 \mathrm{~K}, I_{f}=0.35 \mu \mathrm{A}$ gives the best fit. Subjected to the validity of Eqs. (2) and (3) for the FFO the fits in Fig. 4 support the assumption that additional noise sources (both high and low frequency) were present in the experiments.

Based on numerical simulations it has recently been suggested $^{17}$ that the dynamics of the flux chain moving along the tunnel junction is chaotic at least in certain regions of the parameter space. The fundamental problem is to which extent the small signal sinusoidal ansatz used originally by Nagatsuma et al. ${ }^{1}$ to solve the perturbed sine-Gordon equation is applicable in this very nonlinear system. As pointed out ${ }^{1}$ the magnitude of the junction dissipation, as amply described by the quality factor $Q_{j}$ is of major importance:

$$
Q_{j}=\frac{1}{\left(\frac{f_{j}}{f_{p 0}}\right)^{2} \beta+\alpha}
$$

where $f_{j}=V_{\mathrm{dc}} / \Phi_{0}$ is the Josephson frequency in the bias point, $\beta$ is the surface loss, and $\alpha$ is the shunt loss (in high- $Q_{j}$ junctions usually the quasiparticle loss). A normalized damping $\alpha L / \lambda_{j}>2$ is the accepted condition ${ }^{1,6}$ for the long Josephson tunnel junction to support unidirectional flux flow. The surface loss term usually is neglected in the numerical simulations. The effect of external resistive loading of the FFO has been simulated by Zhang et al. ${ }^{6}$
Despite our FFO junctions being further damped by deposition of an overlaying resistive film (shunting the top and bottom $\mathrm{Nb}$ electrodes) the $Q_{j}$ value even with our relatively high current density is still so large (of order 5-10) that we clearly see the Fiske steps (internal Fiske resonances) superimposed on the FFS, especially at relatively low bias voltages. Also locking to resonant structures in the embedding network could be observed in the $I-V$ curve. The above-mentioned preliminary numerical simulations ${ }^{17}$ showing chaotic dynamics used $\alpha=0.1$, which as mentioned is close to the experimental value for the shunted FFO at low frequencies. Due to the self-inductance of the resistive film this shunting is effective only for frequencies below $200 \mathrm{GHz}$, and so for the linewidth measurements reported here an effective $\alpha=0.02$ seems more appropriate. At frequencies above $400 \mathrm{GHz}$ the surface loss term in Eq. (4) will dominate due to its strong frequency dependence.

From the present measurements of the FFO linewidth we are unable to rule out chaotic dynamics as being the cause for the observed excess noise. Further studies especially as function of temperature may elucidate this very fundamental problem. It should be noted that it still remains to be experimentally proven that the steeping of the FFS (lower $R_{d}$ ) by geometrically injecting currents ${ }^{1}$ into the end of the FFO actually leads to a reduced linewidth. This reduction of the self-field effect in a way shifts the basic problem of dc energy input to the junction from one boundary condition to the other, from dc current injection (usually evenly distributed) along the sides of the unidimensional junction to current injection in the narrow end(s). The latter usually is referred to as "applied magnetic field". 15

In order to be a feasible LO in modern radioastronomical receivers for spectral measurements the linewidth of the FFO must be significantly below $1 \mathrm{MHz}$. For the free-running FFO this seems rather difficult especially at higher frequencies near the superconducting gap. At $f_{\text {FFO }}>500 \mathrm{GHz}$ the surface losses increase considerably and even for "optimal" biasing without self-field effects the differential resistance on the FFS increases, ${ }^{1,6}$ leading to an unacceptable broadening of the linewidth. The FFO in our opinion has superior properties with respect to tuneability, large output power, sinusoidal output with low harmonic content, etc., and we propose to overcome the linewidth problem by phase locking it (e.g., by harmonic injection) to external oscillators or to high$Q$ superconducting on-chip resonators.

\section{HARMONIC PHASE LOCKING}

As described above small Josephson steps of higher order appeared on the $I-V$ curves of both the FFO1 and the SIS detector when a weak signal from the Gunn oscillator was applied to FFO1 despite signals with the fundamental frequency $f_{G}$ of the Gunn oscillator could not propagate along the junction. It means that in certain dc bias ranges the frequency of the FFO1 is phased locked to the $n$th harmonic of the Gunn oscillator. The rf-induced steps did not change their voltage positions with bias cur- 
rent. As a result the linewidth of the FFO1 is given by $\Delta f_{\mathrm{FFO} 1}=n \Delta f_{G}$ (if no other internal linewidth broadening take place in the FFO), and the FFO frequency might be fine tuned by adjusting the frequency $f_{G}$ of the external microwave source.

Figure 5 demonstrates the result of harmonic phase locking. With no applied power from the Gunn oscillator the "usual" mixing of the microwave signals from FFO1 and FFO2 takes place (see curve $A$ ). The corresponding bias point on the autonomous $I-V$ curve of the FFO1 (curve 1) is marked by the arrow $A$ [see inset to Fig. $5(\mathrm{a})]$. In certain frequency ranges the signal from one FFO produces a tiny Shapiro step in the $I-V$ curve of the other and an intermediate frequency signal appears in the band of the IF amplifier whenever the FFO voltage is $\approx 3 \mu \mathrm{V}$ above or below the step. For the results presented in Fig. $5, f_{\mathrm{FFO} 2}$ was equal to $355 \mathrm{GHz}(V=734 \mu \mathrm{V})$ and we used the upper sideband $\left(f_{\mathrm{FFO} 1}=356.5 \mathrm{GHz}\right)$. The soft "bump" seen below point $A$, instead of a fine Shapiro step, is caused by mutual interaction between the two FFO's. The integral linewidth is about $30 \mathrm{MHz}$ (see curve $A$ ) mainly due to the large differential resistance of the FFO1 in the operational point $\left(R_{d} \approx 0.1 \Omega\right)$.

When the microwave signal $\left(f_{G} n=59.415 \mathrm{GHz} \times 6=\right.$ $f_{\mathrm{FFO} 1}=356.5 \mathrm{GHz}$ ) was applied, other Shapiro steps appeared [see arrow $B$ on the pumped curve 2 in Fig. 5(a); note also the transformation of the soft bump into an ordinary Shapiro step in the $I-V$ curve of FFO1 induced by FFO2]. On the rf-induced step caused by harmonics of the Gunn oscillator, the FFO1 is phase locked. As a result the integral linewidth decreases considerably and is determined by FFO2 only. The measured value of the linewidth for the curve $B$ fits with the measured differential resistance of about $0.04 \Omega$ determined from the $I-V$ curve of the FFO2.

In order to verify the absence of any additional broadening of the FFO2 linewidth due to contributions from the locked FFO1 we also measured the linewidth of the FFO2 by direct mixing $f_{\mathrm{FFO} 2}$ with the sixth harmonic of the Gunn oscillator, biasing FFO1 at a considerably lower frequency where it did not take part in the mixing (see curve $C$ and arrow $C$ on curve 2). One can see that the linewidth for the curves $B$ and $C$ is the same, but the power in the first case is $10 \mathrm{~dB}$ larger due to presence of the synchronized signal from FFO1. The curves marked $B$ are essentially similar in Figs. 5(a) and $5(\mathrm{~b})$ (note the

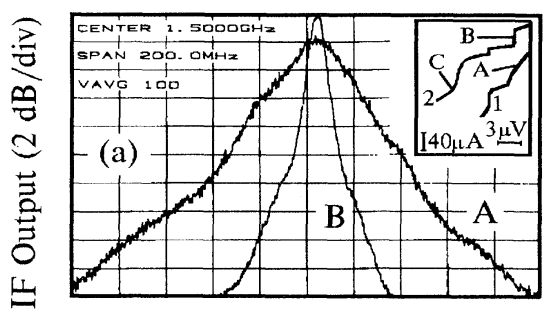

Frequency $(20 \mathrm{MHz} / \mathrm{div})$

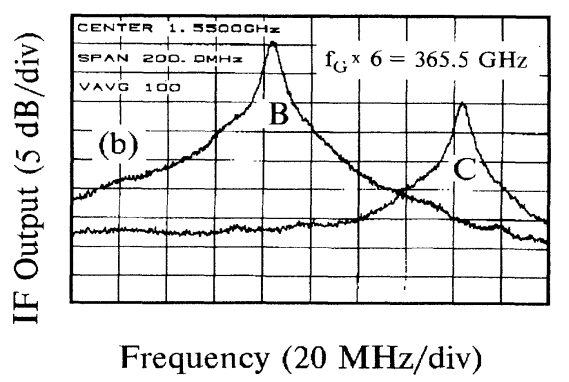

FIG. 5. Spectra of IF power recorded when the signal from FFO2 $(f=355 \mathrm{GHz})$ is mixed with the signal from the autonomous FFO1 (curve $A$ ), the phase-locked FFO1 (curve $B$ ), and the sixth harmonic of the Gunn oscillator (curve $C$ ). The inset shows the corresponding $I-V$ curves of the FFO1: (1) autonomous $I V$ curve and (2) pumped $I-V$ curve (see text).

change in scale from $2 \mathrm{~dB} / \mathrm{div}$ to $5 \mathrm{~dB} / \mathrm{div}$ and the shift in the center frequency).

\section{ACKNOWLEDGMENTS}

The authors thank G. M. Fischer, Britt H. Larsen, S. V. Shitov, and A. V. Ustinov for fruitful discussions and L. V. Filippenko for help with the junction preparation. The work was supported in parts by the NATO Linkage Programme (Grant No. 921040), the Russian Program of Fundamental Research (Contract No. 9202-3484), and the Russian State Scientific Program "Superconductivity" (Contract No. 91009).
${ }^{1}$ T. Nagatsuma, K. Enpuku, F. Irie, and K. Yoshida, J. Appl. Phys. 54, 3302 (1983); J. Appl. Phys. 56, 3284 (1984); 58, 441 (1985); 63, 1130 (1988).

2 A. V. Ustinov, J. Mygind, and V. A. Oboznov, J. Appl. Phys. 72, 1203 (1992).

${ }^{3}$ A. V. Ustinov, T. Doderer, R. P. Huebener, J. Mygind, V. A. Oboznov, and N. F. Pedersen, IEEE Trans. Appl. Supercond. AS-3, 2287 (1993).

${ }^{4}$ V. P. Koshelets, A. V. Shchukin, S. V. Shitov, and L. V. Filippenko, IEEE Trans. Appl. Supercond. AS-3, 2524 (1993).
${ }^{5}$ V. P. Koshelets, A. V. Shchukin, S. V. Shitov, L. V. Filippenko, G. M. Fisher, and J. Mygind, Physica B 194-196, 81 (1994).

${ }^{6}$ Y. M. Zhang, D. Winkler, and T. Claeson, Appl. Phys. Lett. 623195 (1993) and (unpublished).

${ }^{7}$ B. Bi, S. Han, and J. E. Lukens, IEEE Trans. Appl. Superconduct. AS-3, 2303 (1993).

${ }^{8}$ V. K. Kaplunenko, Britt H. Larsen, J. Mygind, and N. F. Pedersen, J. Appl. Phys. 76, 3172 (1994).

${ }^{9}$ S. Han, B. Bi, W. Zhang, and J. E. Lukens, Appl. Phys. Lett. 64, 1424 (1994). 
${ }^{10}$ S. P. Benz and C. J. Burroughs, Appl. Phys. Lett. 58, 2162 (1994).

${ }^{11}$ P. A. A. Booi and S. P. Benz, Appl. Phys. Lett. 64, 2163 (1994).

12 An. B. Ermakov, V. P. Koshelets, S. A. Kovtonjuk, and S. V. Shitov, IEEE Trans. Magn. MAG-27, 2642 (1991).

${ }^{13}$ V. P. Koshelets, S. A. Kovtonyuk, I. L. Serpuchenko, L. V. Filippenko, and A. V. Shchukin, IEEE Trans. Magn. MAG-27, 3141 (1991).

${ }^{14}$ V. P. Koshelets, S. V. Shitov, A. V. Shchukin, L. V. Filip- penko, I. L. Lapytskaya, and J. Mygind, in Applied Superconductivity Conference, Boston, 1994 [IEEE Trans. Appl. Supercond. (to be published)].

${ }^{15}$ K. K. Likharev, Dynamics of Josephson Junctions and Circuits (Gordon and Breach Science Publishers, New York, 1986).

${ }^{16}$ E. Joergensen, V. P. Koshelets, R. Monaco, J. Mygind, M. R. Samuelsen, and M. Salerno, Phys. Rev. Lett. 49, 1093 (1982).

${ }^{17}$ M. R. Samuelsen and M. Salerno (private communication). 